\title{
An Objective Approach to Identify Spectral Distinctiveness for Hearing Impairment
}

\author{
Yeou-Jiunn Chen ${ }^{1}$ and Jiunn-Liang $\mathrm{Wu}^{2}$ \\ ${ }^{1}$ Department of Electrical Engineering, Southern Taiwan University of Science and Technology, Tainan 710, Taiwan \\ ${ }^{2}$ Department of Otolaryngology, College of Medicine, National Cheng Kung University, Tainan 701, Taiwan \\ Correspondence should be addressed to Yeou-Jiunn Chen; chenyj@mail.stust.edu.tw
}

Received 14 September 2013; Accepted 10 October 2013

Academic Editor: Teen-Hang Meen

Copyright (c) 2013 Y.-J. Chen and J.-L. Wu. This is an open access article distributed under the Creative Commons Attribution License, which permits unrestricted use, distribution, and reproduction in any medium, provided the original work is properly cited.

To facilitate the process of developing speech perception, speech-language pathologists have to teach a subject with hearing loss the differences between two syllables by manually enhancing acoustic cues of speech. However, this process is time consuming and difficult. Thus, this study proposes an objective approach to automatically identify the regions of spectral distinctiveness between two syllables, which is used for speech-perception training. To accurately represent the characteristics of speech, mel-frequency cepstrum coefficients are selected as analytical parameters. The mismatch between two syllables in time domain is handled by dynamic time warping. Further, a filter bank is adopted to estimate the components in different frequency bands, which are also represented as mel-frequency cepstrum coefficients. The spectral distinctiveness in different frequency bands is then easily estimated by using Euclidean metrics. Finally, a morphological gradient operator is applied to automatically identify the regions of spectral distinctiveness. To evaluate the proposed approach, the identified regions are manipulated and then the manipulated syllables are measured by a close-set based speech-perception test. The experimental results demonstrated that the identified regions of spectral distinctiveness are very useful in speech perception, which indeed can help speech-language pathologists in speechperception training.

\section{Introduction}

Hearing loss would seriously degrade a subject's speech perception, thereby affecting the development of articulation ability. It then reduces speech intelligibility and affects speech and language development, learning, and communication. Recently, assistive listening devices such as hearing aids or cochlear implants could help subjects with hearing loss utilize their residual hearing and develop their speech perception [1-7]. To facilitate this process, speech-language pathologists (SLPs) have to provide speech-perception training that could increase subject's ability to distinguish one syllable from another. In clinical practice, SLPs manually enhance the distinguishable acoustic cues and extensively use them in speech-perception training. However, it is a time-consuming and expensive process. Thus, it is beneficial for SLPs in speech-perception training if the regions of spectral distinctiveness between two syllables can be identified automatically.
When a speech wave propagates on the basilar membrane, it is characterized as time-spectral patterns. Then, unique perceptual cues, which are the basic units for speech perception, can be identified. Therefore, the relation between the acoustic cues and perceptual units is a key problem for speech perception [8-10]. In the last decade, the relation had been examined [11-18] and the results show that the main factors of acoustic cues are duration, stress, and spectral distinctiveness.

SLPs generally increase the duration and stress of a syllable to teach a subject how to distinguish one syllable from another. Duration and stress can be simply manipulated by speech techniques [19]. However, the spectral distinctiveness between two syllables is very difficult to be identified. In clinical practice, SLPs have to repeatedly pronounce a syllable by enhancing the volume of part segment of a syllable. However, it is a complicated task for SLPs to manually enhance spectral distinctiveness of a syllable. In order to 
identify the regions of spectral distinctiveness, Li et al. proposed a psychoacoustic method in three dimensions: time, frequency, and intensity [20]; still it is a time-consuming process. Moreover, it is difficult to apply to other languages. Hence, to automatically identify the regions of spectral distinctiveness is very important for hearing impairment in speech-perception training.

In this study, an objective approach to identify the regions of spectral distinctiveness is proposed. The mel-frequency cepstrum coefficients (MFCCs) are selected as analytical parameters and used to represent the characteristics of acoustic signal. The mismatch between two syllables in time domain is handled by dynamic time warping; thereby, an optimal matching condition could be obtained. To accurately estimate the spectral similarity, filter bank is applied to find the spectral components of different frequency bands. For the speech signal in each frequency band, the MFCCs are also extracted to represent the acoustical characteristics. According to the optimal matching condition, the spectral distinctiveness of each frequency band between two syllables can be estimated easily by using Euclidean metrics. Finally, the morphological gradient operator is developed to automatically identify the regions of spectral distinctiveness. Moreover, in order to evaluate the accuracy of identified regions of spectral distinctiveness, an acoustic cue manipulation is proposed in this study.

The rest of this paper is organized as follows. Section 2 describes the objective approach to identify spectral distinctiveness including feature extraction, spectral distinctiveness estimation, and spectral distinctiveness identification. Besides, the acoustic cue manipulation is introduced. Section 3 then describes a series of experiments to examine the performance of our approach. Finally, conclusions are drawn in Section 4, along with recommendations for future research.

\section{Materials and Methods}

In this section, the proposed objective approach to identify the regions of spectral distinctiveness between two syllables (as shown in Figure 1) is introduced. Firstly, the MFCCs are extracted from the input speech signals and the filtered speech signals. Secondly, the distance between the MFCCs of two syllables is computed and used to find the consonantvowel boundary. This approach also adopts the dynamic time warping to find an optimal matching condition between two input syllables. Thirdly, according to the optimal matching condition, the spectral distinctiveness of each frequency band can be easily estimated by using Euclidean metric. Finally, a morphological gradient operator is applied to automatically identify the regions of spectral distinctiveness. To examine the proposed approach, an acoustic cues manipulation is also proposed to manipulate the regions of spectral distinctiveness. These procedures are illustrated serially in the following subsections.

2.1. Feature Extraction. Analytical parameters which can accurately represent a speech signal play an important role in objective measurement of spectral distinctiveness. Since, MFCCs had been widely used in speech processing, especially speech recognition [21], they are very suitable for accurately representing not only a speech signal but also speech signals in different frequency bands. The procedure to extract MFCCs from a speech signal is illustrated as follows:

(1) taking the Fourier transform of frames windowed from input speech signal;

(2) mapping the powers of the spectrum onto the mel scale which is defined as

$$
m=2595 \log _{10}\left(1+\frac{h}{700}\right),
$$

where $h$ is the frequency $(\mathrm{Hz})$ in linear domain;

(3) using triangular overlapping windows to get the power spectrum in mel scale;

(4) taking the logs of the powers at each of the mel frequencies which is denoted as $f$;

(5) taking the discrete cosine transform of the mel log powers which is defined as

$$
c(u)=\alpha(u) \sum_{x=0}^{N-1} f(x) \cos \left(\frac{\pi(2 x+1) u}{2 N}\right),
$$

where $N$ is the length of window size and $\alpha(u)$ is defined as

$$
\alpha(u)= \begin{cases}\sqrt{\frac{1}{N}}, & \text { for } u=0 ; \\ \sqrt{\frac{2}{N}}, & \text { for } u \neq 0 ;\end{cases}
$$

(6) finally, MFCCs are composed of amplitudes of the resulting spectrum, $c(u)$.

In Mandarin, a syllable can be decomposed into an INITIAL and a FINAL. INITIALs consist of consonants or semivowels, and FINALs consist of vowels or vowels plus one of the two nasal sounds. Thus, two syllables represented as $s_{i}=c_{i} v$ and $s_{j}=c_{j} v$ are used to estimate the regions of spectral distinctiveness. Let $O_{i}=o_{i 1} o_{i 2} o_{i 3}, \ldots, o_{i n_{i}}$ and $O_{j}=$ $o_{j 1} o_{j 2} o_{j 3}, \ldots, o_{j n_{j}}$, respectively, represent the MFCCs of $s_{i}$ and $s_{j}$, in this study.

In addition, a filter bank is adopted to separate the input signal into multiple components, which are the acoustical characteristics of frequency bands. For the $k$ th frequency band, the corresponding MFCCs of $s_{i}$ and $s_{j}$ are also extracted and denoted as $O_{i}^{k}=o_{i 1}^{k} o_{i 2}^{k} o_{i 3}^{k}, \cdots, o_{i n_{i}}^{k}$ and $O_{j}^{k}=$ $o_{j 1}^{k} o_{j 2}^{k} o_{j 3}^{k}, \cdots, o_{j n_{j}}^{k}$, respectively.

2.2. Spectral Distinctiveness Estimation. In order to estimate the spectral distinctiveness between two syllables, the mismatch should be dealt; thereby, the difference in frequency bands can be easily estimated by using Euclidean metric. Therefore, the dynamic time warping algorithm is adopted to 


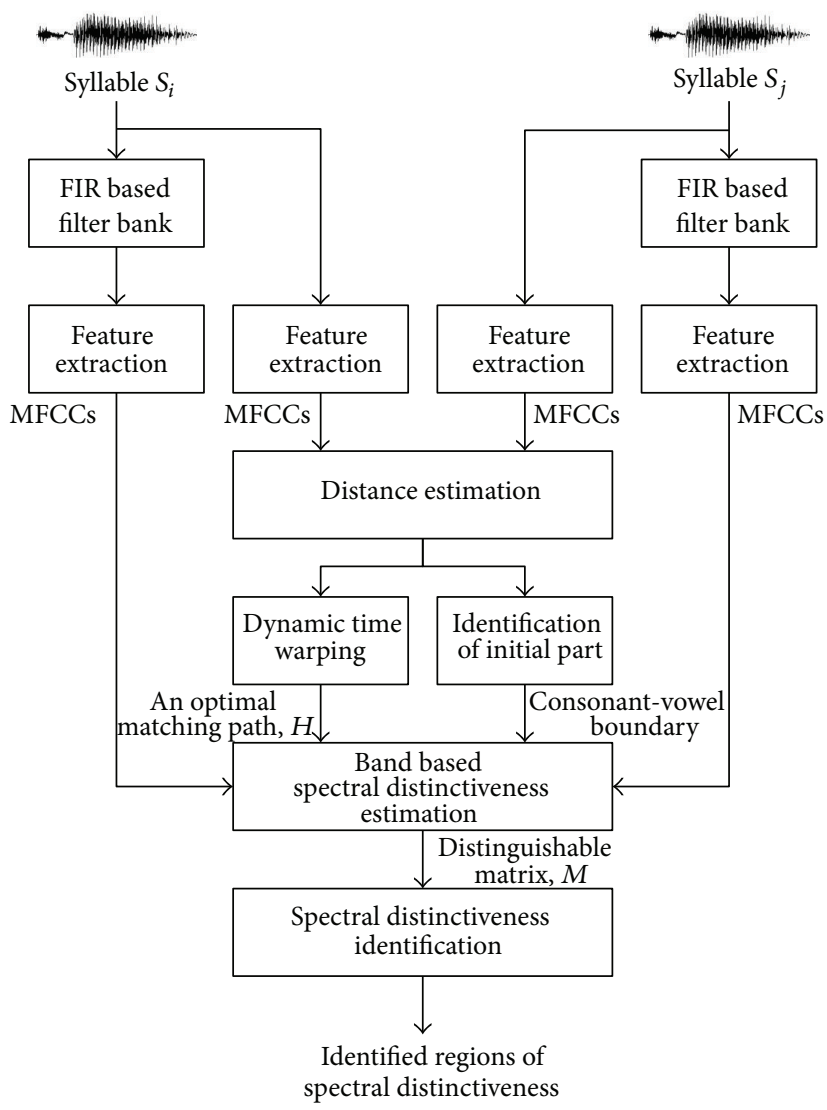

FIGURE 1: The flowchart of the objective approach to identify the regions spectral distinctiveness.

compare two sequences $O_{i}$, and $O_{j}$; thereby, a plane spanned by $O_{i}$ and $O_{j}$ is considered as a distance matrix $D$, which is written as

$$
D\left(O_{i}, O_{j}\right)=\left[\begin{array}{ccc}
d_{11} & \cdots & d_{1 n_{j}} \\
\vdots & \ddots & \vdots \\
d_{n_{i} 1} & \cdots & d_{n_{i} n_{j}}
\end{array}\right]
$$

where $d_{q r}$ is the Euclidean distance between $o_{i q}$ and $o_{j r}$. The matching condition indicating the correspondence between the time axes of $O_{i}$ and $O_{j}$ can be represented a sequence of lattice points on the plane $D$ and written as

$$
H=\left\{\left(h_{1}^{i}, h_{1}^{j}\right),\left(h_{2}^{i}, h_{2}^{j}\right),\left(h_{3}^{i}, h_{3}^{j}\right), \ldots,\left(h_{n}^{i}, h_{n}^{j}\right)\right\},
$$

where $h_{r}^{q}$ is the $r$ th matching pair in $s_{q}$. The $H$ is the best matching condition and then the dynamic time warping algorithm is described as shown in Algorithm 1.

In the dynamic time warping algorithm, the variable is used to store a path, which reaches at lattice point (a, b) with minimum accumulative distance. The minimum accumulative distance is stored in variable DTW $(a, b)$. Since the durations are quite different for each INITIAL, the boundary condition should be ignored in this study. Hence, the path of the lattice point at first row and column goes through its previous lattice point at the step 1 . In order to stop the backtrack for finding the optimal path, path $(1,1)$ is set to be $(0,0)$. In addition, monotony and continuity condition is applied to be concerned in the matching condition at step 2 . It means that the search space of lattice point $(a, b)$ includes three lattice points: $(a-1, b),(a-1, b-1)$ and $(a, b-1)$. At step 3, a simple method is implemented to find an optimal matching condition $H$ by backtracking from lattice point $\left(n_{i}, n_{j}\right)$.

According to the optimal matching condition $H$ and a distinguishable matrix for syllable $s_{i}, M\left(s_{i}\right)$, can be estimated as

$$
M\left(s_{i}\right)=\left[\begin{array}{ccccc}
m_{1}^{F} & m_{2}^{F} & \cdots & m_{n_{-}-1}^{F} & m_{n_{i}}^{F} \\
m_{1}^{F-1} & m_{2}^{F-1} & \cdots & m_{n_{i}-1}^{F-1} & m_{n_{i}}^{F-1} \\
\vdots & \vdots & \ddots & \vdots & \vdots \\
m_{1}^{2} & m_{2}^{2} & \cdots & m_{n_{i}-1}^{2} & m_{n_{i}}^{2} \\
m_{1}^{1} & m_{2}^{1} & \cdots & m_{n_{i}-1}^{1} & m_{n_{i}}^{1}
\end{array}\right]
$$

where $F$ is the number of filter bands and $m_{t}^{f}$ is the distance for frame $t$ at $f$ th frequency band. $m_{t}^{f}$ can be defined as

$$
m_{t}^{f}=\frac{1}{\# c} \sum_{c \ni(t, c) \in H} \operatorname{Dist}\left(o_{i t}^{f}, o_{j c}^{f}\right),
$$

where \#c is the number of frames in $O_{j}$ which is matched with frame $t$ in $O_{i}$. 
Step 1. Initialization

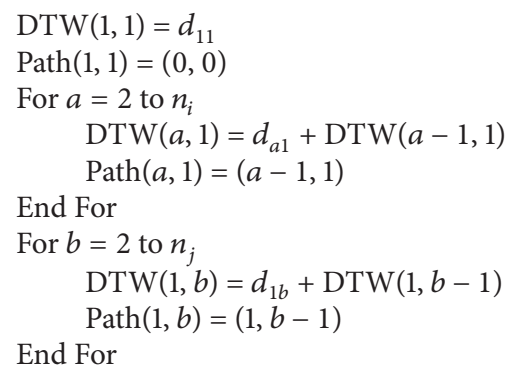

Step 2. Iteration

For $a=2$ to $n_{i}$ For $b=2$ to $n_{j}$

$$
\begin{aligned}
& \operatorname{DTW}(a, b)=d_{a b}+\underset{v \in\{((a-1), b),(a,(b-1)),((a-1),(b-1))\}}{\arg \min }\left\{d_{v}\right\} \\
& \operatorname{Path}(a, b)=\underset{v \in\{((a-1), b),(a,(b-1)),((a-1),(b-1))\}}{ }\left\{d_{v}\right\}
\end{aligned}
$$

End For

End For

Step 3. Backtracking and Termination

The optimal (minimum) distance is $\operatorname{DTW}\left(n_{i}, n_{j}\right)$.

The optimal matching path $H$ is found by simple backtracking from $\operatorname{Path}\left(n_{i}, n_{j}\right)$.

$H=\varnothing$

$l p=\left(n_{i}, n_{j}\right)$

While $l p \neq(0,0)$ Do

$H=H \cup l p$

$l p=\operatorname{Path}(l p)$

End While

Algorithm 1: The dynamic time warping algorithm.

2.3. Spectral Distinctiveness Identification. The spectral distinctiveness between two syllables is estimated; thereby, the regions of spectral distinctiveness should be identified from the distinguishable matrix $M$. Recently, the grayscale morphological gradient operator is a powerful and fast technique for both contour detection and region based segmentation [22]. Thus, it can be successfully used to detect the regions of spectral distinctiveness. To obtain the regions of spectral distinctiveness from $M$, the morphological gradient operator is used and defined as

$$
M G(f)=\frac{1}{n} \sum_{i=1}^{n}\left(\left(\left(f \oplus b_{i}\right)-\left(f \Theta b_{i}\right)\right) \oplus b_{i-1}\right),
$$

where $n$ is the scale of morphological gradient operator and $b_{i}$ denotes the group of square structuring elements.

In (8), the symbols $\oplus$ and $\Theta$ are the grayscale dilation and grayscale erosion, which are defined as follows:

$$
\begin{aligned}
& (M \oplus b)(s, t) \\
& =\max \left(M(s-x, t-y)-\frac{b(x, y)}{(s-x)}\right. \\
& \left.\quad \mid(t-y) \in D_{f},(s, y) \in D_{b}\right),
\end{aligned}
$$

$$
\begin{aligned}
& (M \Theta b)(s, t) \\
& =\max \left(M(s-x, t+y)-\frac{b(x, y)}{(s+x)}\right. \\
& \left.\quad \mid(t+y) \in D_{f},(s, y) \in D_{b}\right),
\end{aligned}
$$

where $b$ is a flat structuring element. $D_{f}$ and $D_{b}$ are the domains of $M$ and $b$, respectively. According to (9), the grayscale opening and closing then can be derived as

$$
\begin{aligned}
& f \circ b=(f \Theta b) \oplus b, \\
& f \cdot b=(f \oplus b) \Theta b .
\end{aligned}
$$

2.4. Acoustic Cues Manipulation. The accuracy of identify regions of spectral distinctiveness was examined in this subsection. Therefore, the power of the spectrogram of these regions should be manipulated to examine that a syllable is converted to another. Thus, a speech modification procedure based on short-time Fourier transform (STFT) is proposed to analyze a speech sound and then synthesize an enhanced speech sound [23]. 


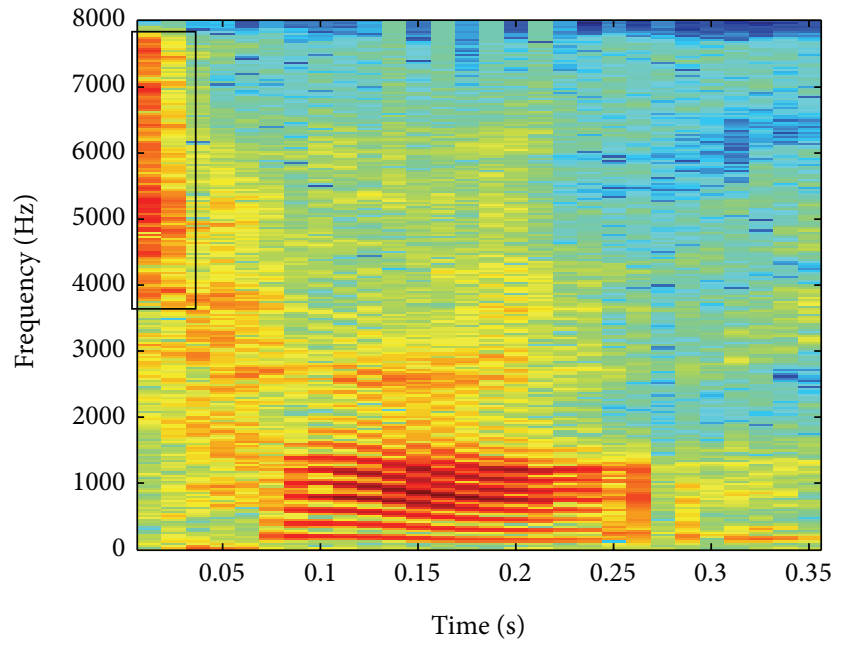

(a)

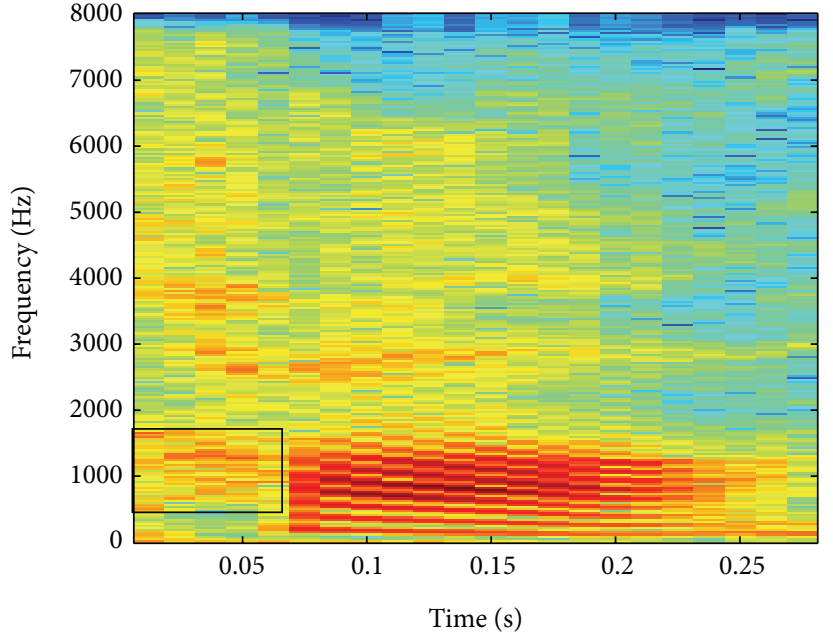

(b)

Figure 2: The spectrograms of (a) /ta/ and (b) /ka/.

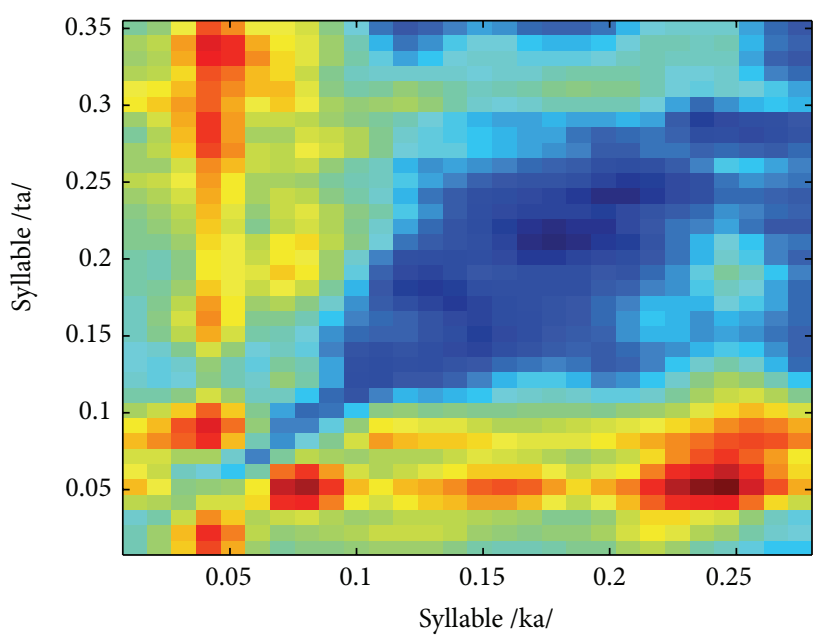

(a)

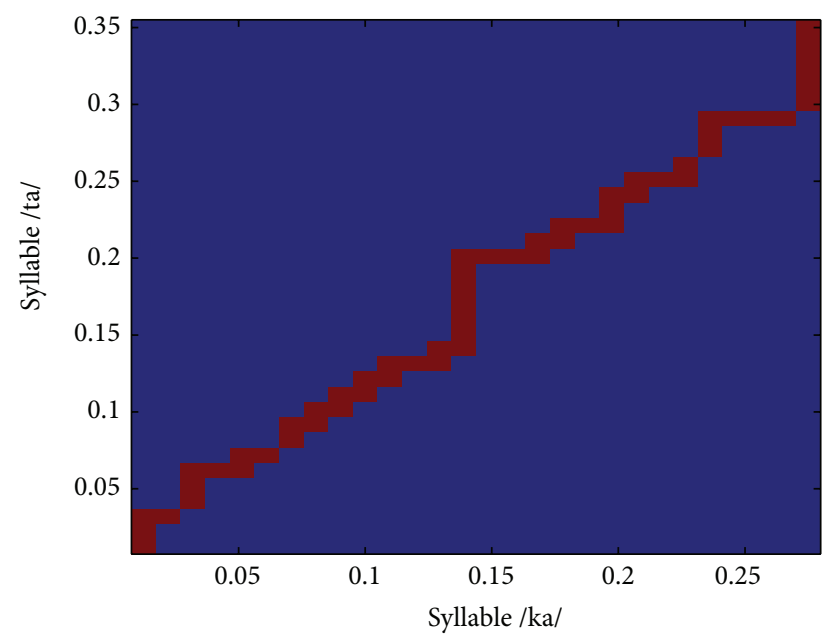

(b)

FIgURE 3: The results of (a) distance matrix and (b) optimal matching condition.

Let $x[n]$ denote the speech signal at sample times $n$. A Hamming window $w[n]$ is used to divide $x[n]$ into $N$ point overlapping frames, which can be written as

$$
x[m, n] \equiv w[n] x[m R-n],
$$

where $R$ is the step size and is defined as $N / 4$. Therefore, the resulting STFT coefficients $X[m, k]$ can be derived as

$$
X[m, k]=\sum_{n=0}^{N-1} x[m, n] e^{-j 2 \pi k n / N} .
$$

To improve the accuracy of modification, the windowed speech is zero-padded before performing the Fourier transform.

The region of spectral distinctiveness is then modified by multiplying a specific gain $M[m, k]$. Specifically, $M[m, k]=0$ indicates feature removal, $0<M[m, k]<1$ corresponds to feature attenuation, and $M[m, k]>1$ represents feature enhancement. Thus, the modified speech spectrum can be written as

$$
\widetilde{X}[m, k]=X[m, k] \cdot M[m, k] .
$$

Generally, the gain is expressed in $\mathrm{dB}$ as

$$
G=20 \log _{10}(M[m, k]) .
$$

According to $\widetilde{X}[m, k]$, the single frame signal is recovered by applying an inverse Fourier transform, which is defined as follow:

$$
\widetilde{x}[m, n]=\frac{1}{N} \sum_{k=0}^{N-1} \widetilde{X}[m, k] e^{j 2 \pi k n / N}
$$




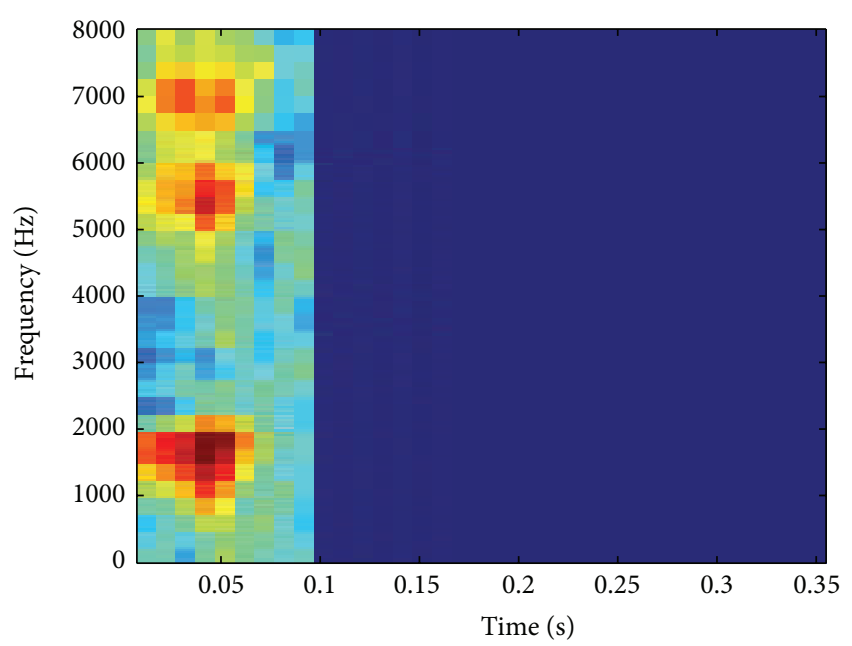

(a)

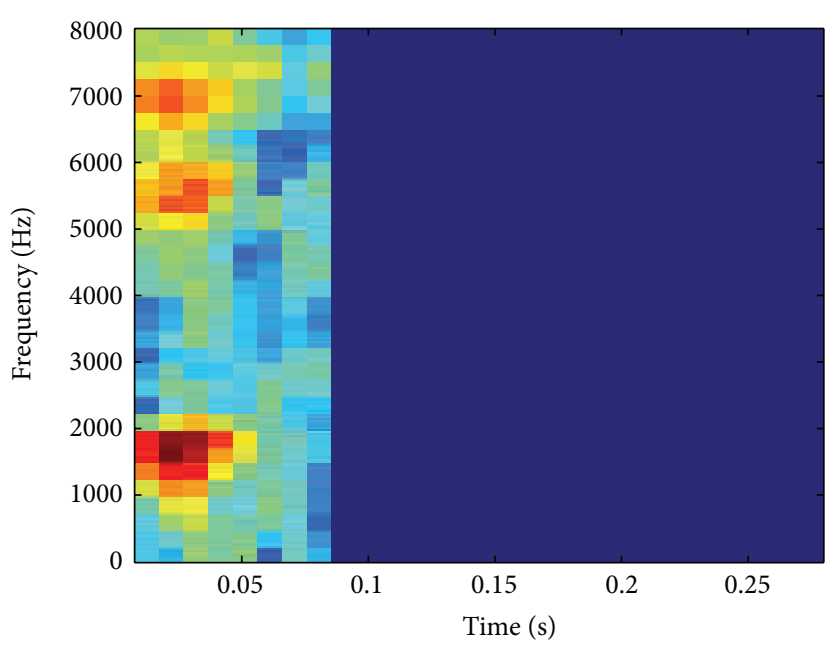

(b)

FIgURE 4: The results of distinguishable matrix for (a)/ta/ and (b) /ka/.

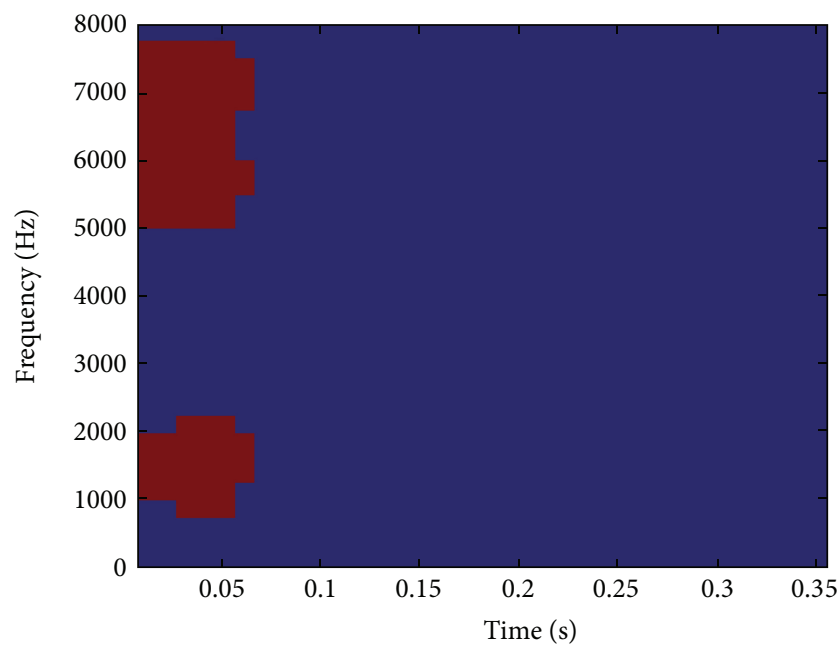

(a)

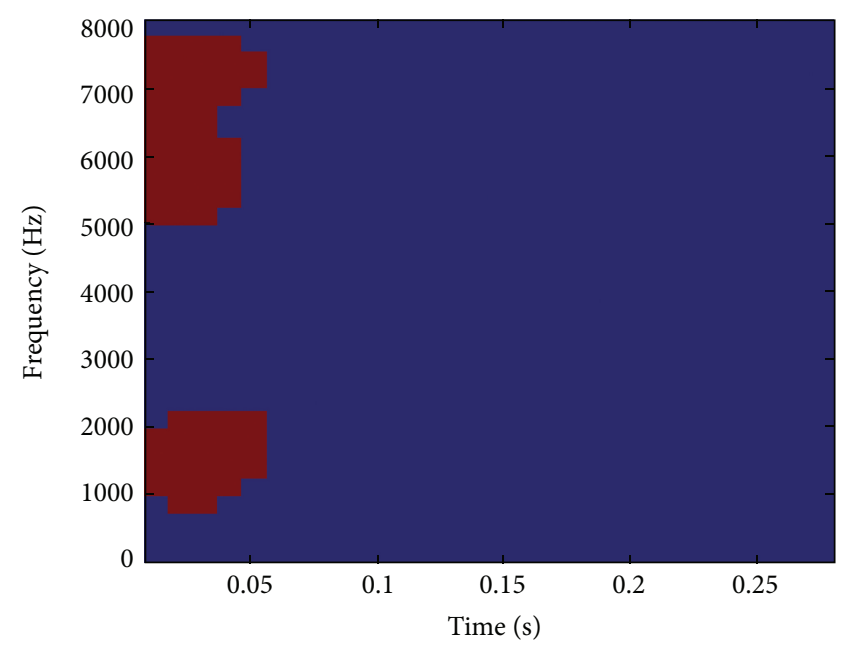

(b)

Figure 5: The identified regions of spectral distinctiveness for (a)/ta/ and (b)/ka/.

Finally, an overlap-add synthesis is used to generate the modified speech $\widetilde{X}[n]$ in time domain, which can be written as

$$
\widetilde{X}[n]=\sum_{m=-M_{0}}^{0} \widetilde{X}[m R, n] .
$$

\section{Results and Discussions}

To evaluate the proposed approach, a close-set based speechperception test on stop consonants was performed in this study. The speech stimuli, including the syllables /da, ga, ka, $\mathrm{ba}, \mathrm{pa}, \mathrm{ta} /$, were chosen from the University of Pennsylvania's Linguistic Data Consortium (LDC) LDC2005S22. The detailed experimental results are shown as follows.
3.1. Results of Manipulating $/ \mathrm{ta} /$ and $/ \mathrm{ka} /$. In this subsection, the syllables $/ \mathrm{ta} /$ and $/ \mathrm{ka} /$ were used to illustrate the results of the proposed approach. First, the regions of spectral distinctiveness were manually identified to check the results of our approach. The spectrograms of $/ \mathrm{ta} /$ and $/ \mathrm{ka} /$ were shown in Figure 2. It is obvious that /ta/ has high-frequency burst above $4 \mathrm{k} \mathrm{Hz}$ (marked as black rectangle) and /ka/ has a low-frequency burst about $1 \mathrm{k} \mathrm{Hz}$ (marked as black rectangle). These two regions should be very important to distinguish $/ \mathrm{ta} /$ from $/ \mathrm{ka} /$.

Second, the results of distance matrix and dynamic time warping algorithm are examined here. The distance matrix $D$ estimated from $/ \mathrm{ta} /$ and $/ \mathrm{ka} /$ is shown in Figure 3(a). In this figure, the FINALs of $/ \mathrm{ta} /$ and $/ \mathrm{ka} /$ are the same; then the distances between the speech segments of FINALs are very small. The distances between the speech segments of FINALs 


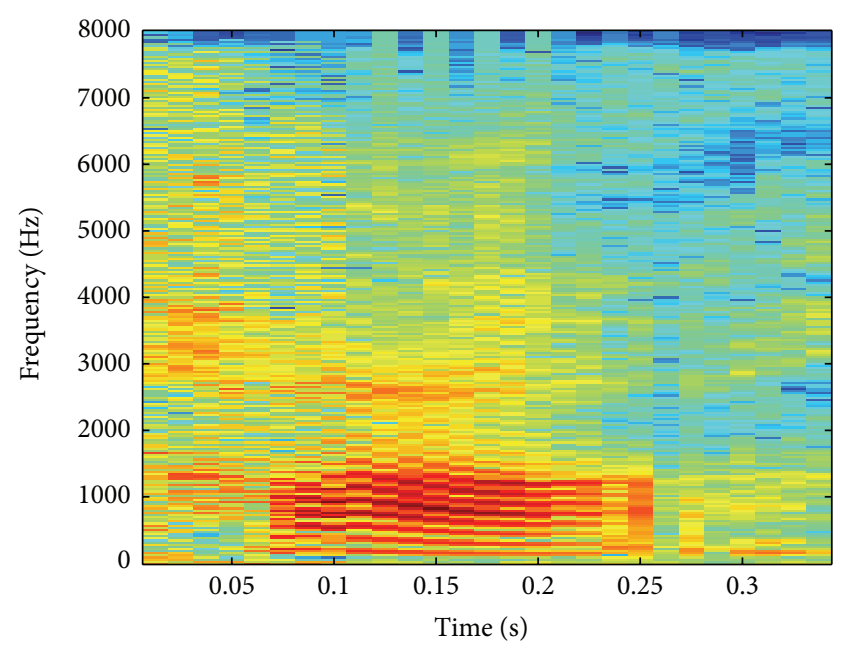

(a)

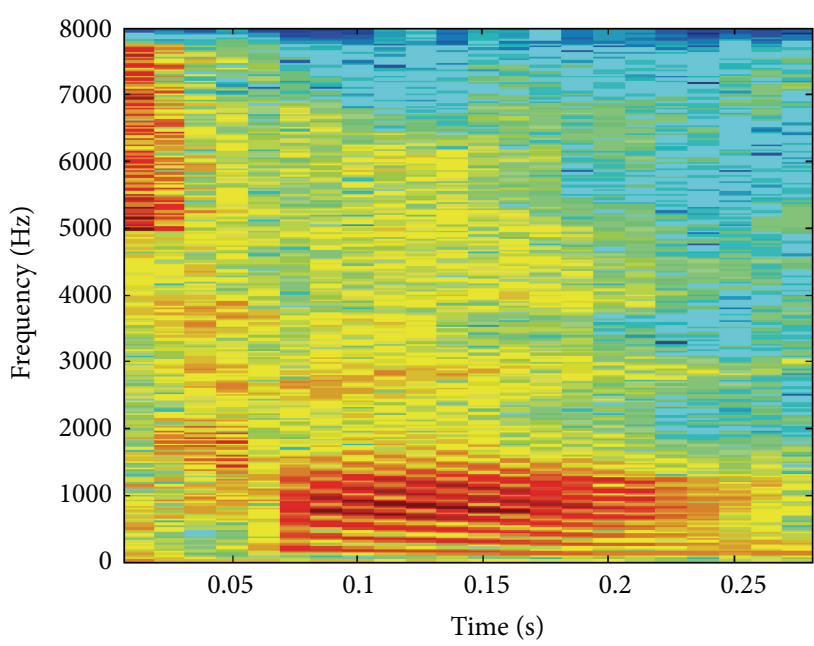

(b)

Figure 6: The spectrograms of manipulated syllables (a) / ta $\rightarrow \mathrm{ka} /$ and (b) / $\mathrm{ka} \rightarrow \mathrm{ta} /$.

and those of INITIALs are much large. Further, the speech segments of INITIALs for $/ \mathrm{ta} / \mathrm{and} / \mathrm{ka} /$ are also quite different. Obviously, there exists an optimal path in 45-degree. In Figure 3(b), the optimal matching path can be successfully detected by dynamic time warping algorithm. It demonstrates that the mismatch between two syllables in time domain can be handled in our approach. Besides, the first frame and the third frame of $/ \mathrm{ka} /$ match with three frames of $/ \mathrm{ta} /$; thus, the first frame and the third frame of $/ \mathrm{ka} /$ should be duplicated three times when $/ \mathrm{ka} /$ is manipulated as $/ \mathrm{ta} /$. It also shows that our approach can be applied to correctly increase the duration of a syllable.

Third, the spectral distinctiveness between $/ \mathrm{ta} /$ and $/ \mathrm{ka} /$ measured by our approach is validated with manually identified results. Therefore, with the optimal matching path (shown in Figure 3(b)), the MFCCs of $/ \mathrm{ta} /$ and $/ \mathrm{ka} / \mathrm{in}$ different frequency bands were adopted to estimate the spectral distinctiveness. The results were normalized in time domain and shown in Figure 4. Comparing Figures 4 and 2, it is obvious that the differences in spectrogram are precisely estimated. By selecting a suitable threshold in morphological gradient operator, the regions of spectral distinctiveness for $/ \mathrm{ta} /$ and $/ \mathrm{ka} / \mathrm{can}$ be identified (shown in Figure 5), which are very similar to the expected regions (shown in Figure 2).

Finally, the regions of spectral distinctiveness should be manipulated to examine the accuracy by a subject. According to the identified regions in Figure 5, the acoustic cues manipulation was applied to modify $/ \mathrm{ta} /$ and $/ \mathrm{ka} / . / \mathrm{ta} /$ and $/ \mathrm{ka} /$ were converted to / ka/ (denoted as $/ \mathrm{ta} \rightarrow \mathrm{ka} /$ ) and $/ \mathrm{ta} /$ (denoted as $/ \mathrm{ka} \rightarrow \mathrm{ta} /$ ), respectively. Then, the spectrograms of /ta $\rightarrow \mathrm{ka} /$ and $/ \mathrm{ka} \rightarrow \mathrm{ta} /$ were shown in Figure 6. Comparing Figure 6(a) with Figure 2(b), the spectral energy about $1 \mathrm{k} \mathrm{Hz}$ had been relatively decreased. Comparing Figure 6(b) with Figure 2(a), the spectral energy above $4 \mathrm{~K}$ increases. Therefore, /ta/ and $/ \mathrm{ka} /$ are heard as $/ \mathrm{ka} /$ and $/ \mathrm{ta} /$, respectively. So, the identified regions really play an important role in distinguishing one syllable from another.
3.2. Experimental Results of Subject Evaluation. In this subsection, the results of manipulated syllables are used to examine the identified regions of spectral distinctiveness. Seven males and three females (college students, age about 30 years) were asked to participate in this study. Each token with and without manipulation was randomly presented to each subject 5 times. The speech stimuli were played at the most comfortable level (around $70 \mathrm{~dB}$ SPL) for the listeners. The parameters of gain $G$ in (14) were set to be $3 \mathrm{~dB}, 6 \mathrm{~dB}$, $9 \mathrm{~dB}$, and $12 \mathrm{~dB}$. After each presentation, subjects responded to the stimulus by clicking on one of two buttons labeled with syllables. The detailed results in recognition rate (\%) are shown in Table 1 . The experimental results show that the recognition rate is over $86 \%$. Moreover, the average recognition rates of manipulated syllables are $89.53 \%, 91.27 \%$, $92.87 \%$, and $92.80 \%$ for $G$ which is $3 \mathrm{~dB}, 6 \mathrm{~dB}, 9 \mathrm{~dB}$, and $12 \mathrm{~dB}$, respectively. When the gain $G$ is set to be $9 \mathrm{~dB}$, it can achieve the best recognition rate. Then, speech intelligibility is distorted for the larger gain.

To objectively compare these results, the syllables without manipulation were also used and the results are shown in Table 2 . The average of recognition rate is $94.93 \%$ which is very similar to that of syllables with manipulation $(G=9 \mathrm{~dB})$. It means that a syllable can be heard as another syllable by manipulating these regions of spectral distinctiveness. Hence, the identified regions of spectral distinctiveness really play an important role in speech perception. SLPs then can apply the identified regions of spectral distinctiveness to help a subject with hearing loss increase his/her ability to distinguish one syllable from another; thereby, the process of speech-perception training then can be facilitated.

\section{Conclusions}

In this study, an objective approach is proposed to identify the regions of spectral distinctiveness between two syllables. The MFCCs are appropriate to represent not only the speech 
TABLE 1: The recognition rates (\%) of syllables with identified regions enhanced by (a) $3 \mathrm{~dB}$, (b) $6 \mathrm{~dB}$, (c) $9 \mathrm{~dB}$, and (d) $12 \mathrm{~dB}$.

(a)

\begin{tabular}{lcccccc}
\hline \multirow{2}{*}{ Manipulated syllable } & \multicolumn{7}{c}{ Target syllable } \\
& da & ga & ka & ba & pa & ta \\
\hline $\mathrm{da}$ & - & 88 & 90 & 90 & 88 & 90 \\
$\mathrm{ga}$ & 92 & - & 94 & 84 & 86 & 88 \\
$\mathrm{ka}$ & 88 & 86 & - & 90 & 92 & 96 \\
$\mathrm{ba}$ & 96 & 96 & 90 & - & 92 & 92 \\
$\mathrm{pa}$ & 84 & 88 & 88 & 86 & - & 90 \\
ta & 90 & 90 & 88 & 86 & 88 & - \\
\hline
\end{tabular}

(b)

\begin{tabular}{lcccccc}
\hline \multirow{2}{*}{ Manipulated syllable } & \multicolumn{7}{c}{ Target syllable } \\
& da & ga & ka & ba & pa & ta \\
\hline $\mathrm{da}$ & - & 88 & 90 & 90 & 88 & 90 \\
ga & 94 & - & 94 & 92 & 96 & 88 \\
$\mathrm{ka}$ & 88 & 96 & - & 90 & 92 & 96 \\
$\mathrm{ba}$ & 96 & 96 & 90 & - & 92 & 94 \\
$\mathrm{pa}$ & 90 & 88 & 88 & 90 & - & 90 \\
$\mathrm{ta}$ & 96 & 90 & 88 & 90 & 88 & - \\
\hline
\end{tabular}

(c)

\begin{tabular}{lcccccc}
\hline \multirow{2}{*}{ Manipulated syllable } & \multicolumn{7}{c}{ Target syllable } \\
& da & ga & ka & ba & pa & ta \\
\hline $\mathrm{da}$ & - & 90 & 92 & 92 & 90 & 92 \\
ga & 94 & - & 94 & 92 & 96 & 92 \\
$\mathrm{ka}$ & 94 & 96 & - & 90 & 94 & 96 \\
$\mathrm{ba}$ & 96 & 96 & 90 & - & 94 & 94 \\
$\mathrm{pa}$ & 90 & 94 & 92 & 90 & - & 92 \\
$\mathrm{ta}$ & 96 & 90 & 94 & 90 & 94 & - \\
\hline
\end{tabular}

(d)

\begin{tabular}{lcccccc}
\hline \multirow{2}{*}{ Manipulated syllable } & \multicolumn{5}{c}{ Target syllable } \\
& da & ga & ka & ba & pa & ta \\
\hline $\mathrm{da}$ & - & 90 & 92 & 92 & 90 & 92 \\
$\mathrm{ga}$ & 94 & - & 94 & 92 & 96 & 92 \\
$\mathrm{ka}$ & 94 & 96 & - & 90 & 92 & 96 \\
$\mathrm{ba}$ & 96 & 96 & 92 & - & 92 & 94 \\
$\mathrm{pa}$ & 90 & 94 & 92 & 90 & - & 92 \\
$\mathrm{ta}$ & 96 & 90 & 94 & 90 & 94 & - \\
\hline
\end{tabular}

signal but also the speech components in different frequency bands. In addition, the use of the dynamic time warping overcomes the mismatch between two speech signals in time domain. According to the optimal matching condition, the spectral distinctiveness of each frequency band between two syllables is easily estimated by using Euclidean metrics. The regions of spectral distinctiveness are precisely identified by morphological gradient operator. The experimental results demonstrate that the identify regions play an important role in distinguishing one syllable from another. In the future, the regions of spectral distinctiveness should be automatically
TABLE 2: Recognition rates (\%) of syllables without manipulation.

\begin{tabular}{lcccccc}
\hline \multirow{2}{*}{ Testing syllable } & \multicolumn{6}{c}{ Competitive syllable } \\
& da & ga & ka & ba & pa & ta \\
\hline $\mathrm{da}$ & - & 94 & 92 & 94 & 92 & 92 \\
$\mathrm{ga}$ & 96 & - & 96 & 96 & 94 & 96 \\
$\mathrm{ka}$ & 94 & 96 & - & 94 & 96 & 94 \\
$\mathrm{ba}$ & 94 & 94 & 92 & - & 98 & 96 \\
$\mathrm{pa}$ & 92 & 96 & 96 & 94 & - & 92 \\
$\mathrm{ta}$ & 96 & 94 & 92 & 92 & 94 & - \\
\hline
\end{tabular}

enhanced and extensively used in speech-perception training; then it can efficiently reduce the loading of SLPs and facilitate the process of developing speech perception.

\section{Acknowledgment}

The authors would like to thank the National Science Council of the Republic of China, Taiwan, for financially supporting this work under Contract NSC 102-2221-E-218-001.

\section{References}

[1] S. Hintea, P. Faragó, M. N. Roman, G. Oltean, and L. Feştilă, "A programmable gain amplifier for automated gain control in auditory prostheses," Journal of Medical and Biological Engineering, vol. 31, no. 3, pp. 185-192, 2011.

[2] P. M. Bollard, P. M. Chute, A. Popp, and S. C. Parisier, "Specific language growth in young children using the claron cochlear implant," The Annals of Otology, Rhinology and Laryngology, vol. 177, pp. 119-123, 1999.

[3] R. T. Vieira, N. Brunet, S. C. Costa, S. Correia, B. G. Aguiar Neto, and J. M. Fechine, "Combining entropy measures and cepstral analysis for pathological voices assessment," Journal of Medical and Biological Engineering, vol. 32, no. 6, pp. 429-436, 2012.

[4] T. A. Meyer, M. A. Svirsky, K. I. Kirk, and R. T. Miyamoto, "Improvements in speech perception by children with profound prelingual hearing loss: effects of device, communication mode, and chronological age," Journal of Speech, Language, and Hearing Research, vol. 41, no. 4, pp. 846-858, 1998.

[5] N. Tye-Murray, L. Spencer, and G. G. Woodworth, "Acquisition of speech by children who have prolonged cochlear implant experience," Journal of Speech and Hearing Research, vol. 38, no. 2, pp. 327-337, 1995.

[6] J. L. Wu and H. M. Yang, "Speech perception of mandarin Chinese speaking young children after cochlear implant use: effect of age at implantation," International Journal of Pediatric Otorhinolaryngology, vol. 67, no. 3, pp. 247-253, 2003.

[7] J. F. Yu, C. K. Chen, S. R. Wang et al., "Activation of the auditory cortex in subjects with unilateral sensorineural hearing impairment in response to hearing their own names," Journal of Medical and Biological Engineering, vol. 30, no. 4, pp. 215-219, 2010.

[8] H. Fletcher and R. Galt, "Perception of speech and its relation to telephony," Journal of the Acoustical Society of America, vol. 22, pp. 89-151, 1950.

[9] J. B. Allen, "Harvey Fletcher's role in the creation of communication acoustics," Journal of the Acoustical Society of America, vol. 99, no. 4 I, pp. 1825-1839, 1996. 
[10] J. B. Allen, Articulation and Intelligibility, Morgan and Claypool, LaPorte, Colo, USA, 2005.

[11] A. Cristià, G. L. McGuire, A. Seidl, and A. L. Francis, "Effects of the distribution of acoustic cues on infants' perception of sibilants," Journal of Phonetics, vol. 39, no. 3, pp. 388-402, 2011.

[12] S. Anand, R. Shrivastav, J. M. Wingate, and N. N. Chheda, "An acoustic-perceptual study of vocal tremor," Journal of Voice, vol. 26, no. 6, pp. 811.e1-811.e7, 2012.

[13] J. E. Lupo, K. Koka, J. L. Thornton, and D. J. Tollin, “The effects of experimentally induced conductive hearing loss on spectral and temporal aspects of sound transmission through the ear," Hearing Research, vol. 272, no. 1-2, pp. 30-41, 2011.

[14] S. Bouton, P. Colé, and W. Serniclaes, "The influence of lexical knowledge on phoneme discrimination in deaf children with cochlear implants," Speech Communication, vol. 54, no. 2, pp. 189-198, 2012.

[15] M. Steinschneider and Y. I. Fishman, "Enhanced physiologic discriminability of stop consonants with prolonged formant transitions in awake monkeys based on the tonotopic organization of primary auditory cortex," Hearing Research, vol. 271, no. 1-2, pp. 103-114, 2011.

[16] T. Kendall and V. Fridland, "Variation in perception and production of mid front vowels in the U.S. southern vowel shift," Journal of Phonetics, vol. 40, no. 2, pp. 289-306, 2012.

[17] I. Grichkovtsova, M. Morel, and A. Lacheret, "The role of voice quality and prosodic contour in affective speech perception," Speech Communication, vol. 54, no. 3, pp. 414-429, 2012.

[18] B. E. Lobdell, J. B. Allen, and M. A. Hasegawa-Johnson, "Intelligibility predictors and neural representation of speech," Speech Communication, vol. 53, no. 2, pp. 185-194, 2011.

[19] Y. C. Huang, C. H. Wu, and Y. T. Chao, "Personalized spectral and prosody conversion using frame-based codeword distribution and adaptive CRF," Proceedings of the IEEE Transactions on Audio, Speech, and Language Processing, vol. 21, no. 1, pp. 51-61, 2013.

[20] F. Li, A. Menon, and J. B. Allen, "A psychoacoustic method to find the perceptual cues of stop consonants in natural speech," Journal of the Acoustical Society of America, vol. 127, no. 4, pp. 2599-2610, 2010.

[21] Y. J. Chen, "Identification of articulation error patterns using a novel dependence network," IEEE Transactions Biomedical Engineering, vol. 58, no. 11, pp. 3061-3068, 2011.

[22] K. Parvati, B. S. Prakasa Rao, and M. M. Das, "Image segmentation using gray-scale morphology and marker-controlled watershed transformation," Discrete Dynamics in Nature and Society, vol. 2008, Article ID 384346, 8 pages, 2008.

[23] Y. J. Chen and J. L. Wu, "A novel speech enhancement using forward-backward minima-controlled recursive averaging," Journal of the Chinese Institute of Engineers, 2013. 


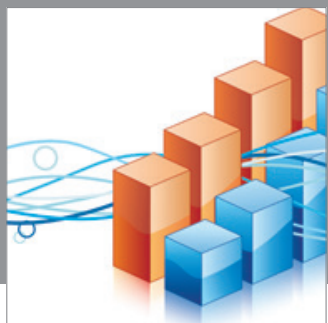

Advances in

Operations Research

mansans

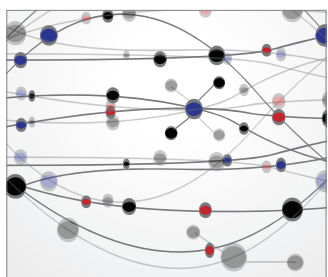

The Scientific World Journal
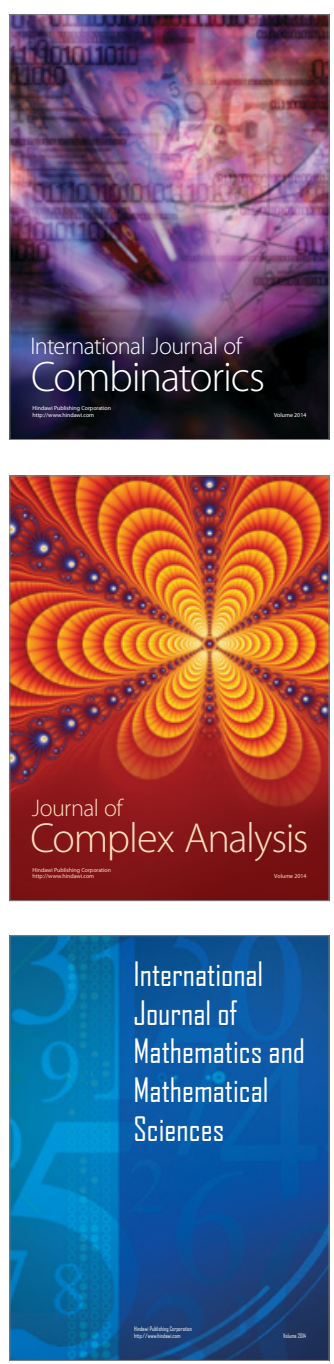
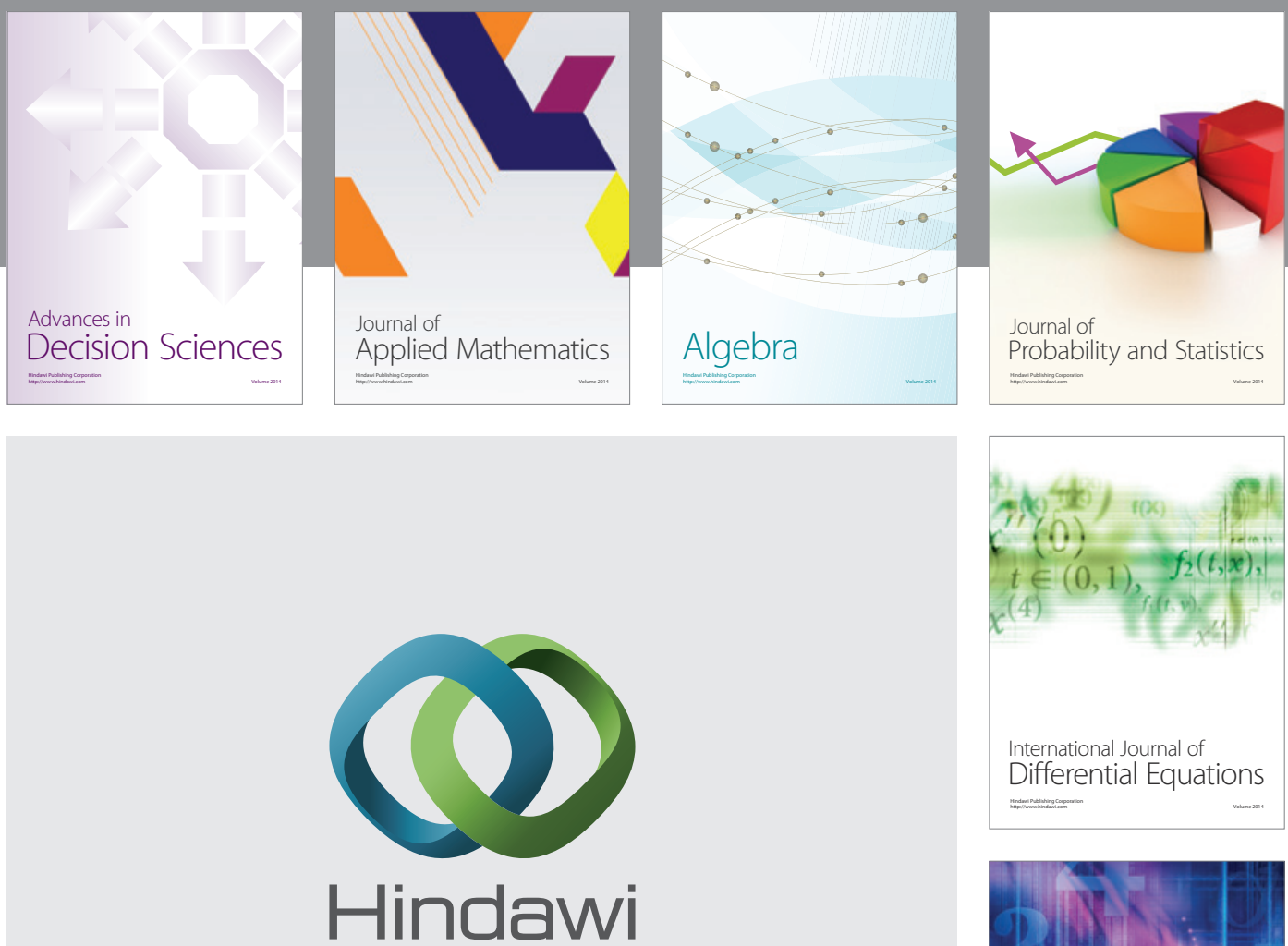

Submit your manuscripts at http://www.hindawi.com
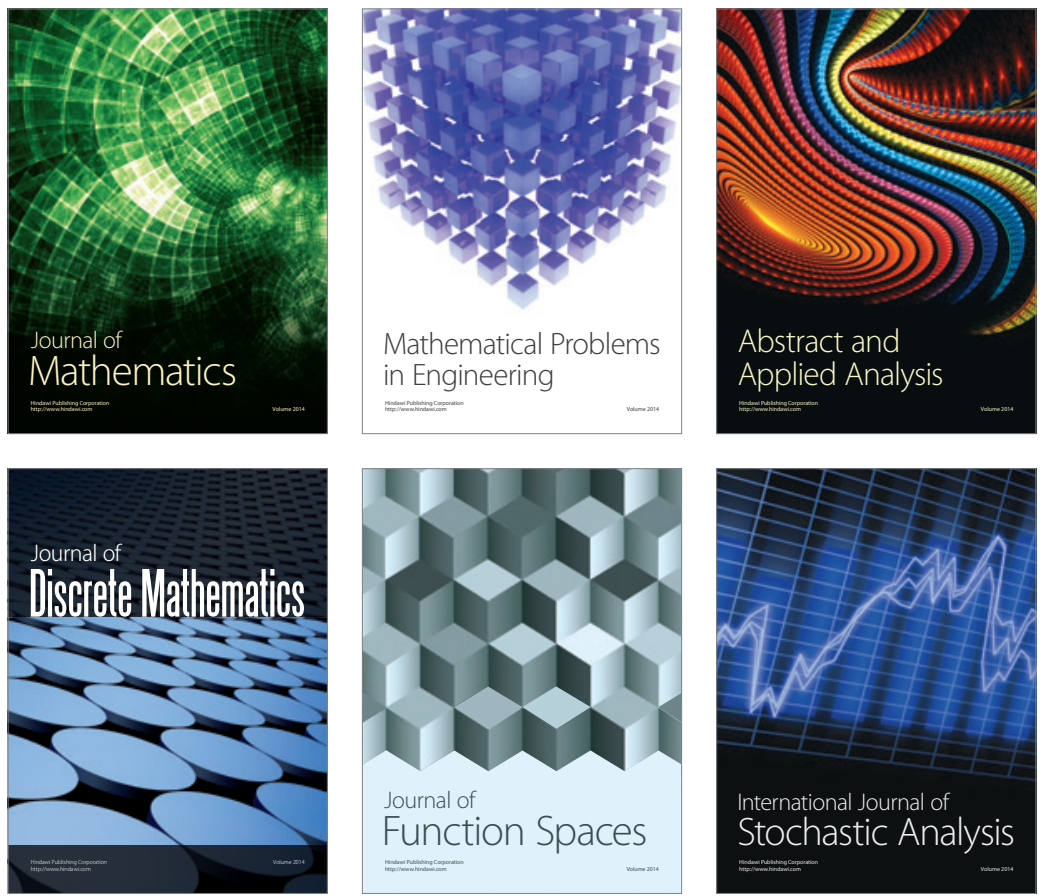

Journal of

Function Spaces

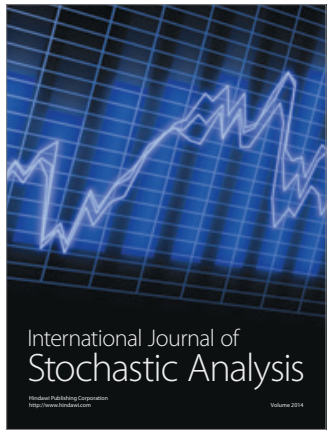

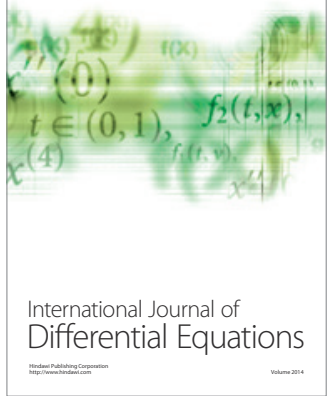
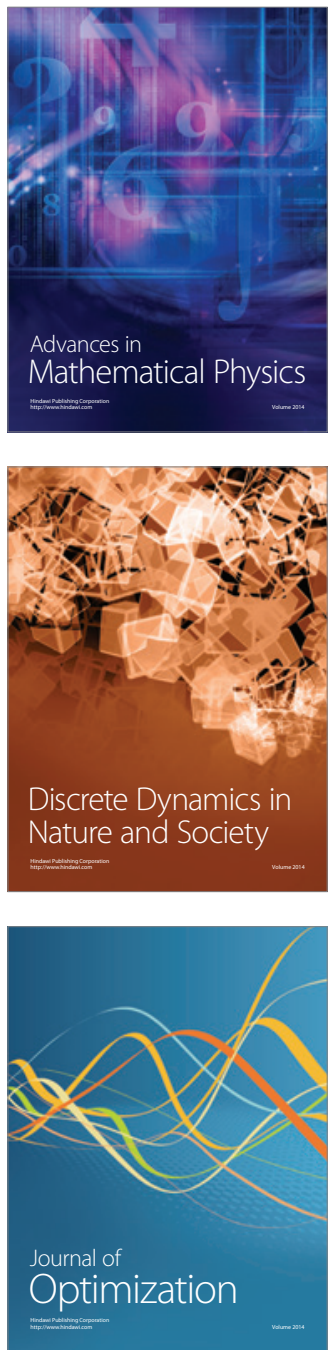